\title{
Statistical Analysis of Brain Natriuretic Peptide in the Treatment of Heart Failure
}

\author{
Yizeng Li ${ }^{1}$, Hong Zhang ${ }^{2}$ \\ ${ }^{1}$ Mathematics and Applied Mathematics, Hangzhou Dianzi University, Zhejiang, China \\ ${ }^{2}$ School of Information, Beijing Wuzi University, Beijing, China
}

Email address:

dr.yuwenjunxian@gmail.com (Hong Zhang)

\section{To cite this article:}

Yizeng Li, Hong Zhang. Statistical Analysis of Brain Natriuretic Peptide in the Treatment of Heart Failure. American Journal of Clinical and Experimental Medicine. Vol. 3, No. 5, 2015, pp. 222-227. doi: 10.11648/j.ajcem.20150305.14

\begin{abstract}
In the clinical researches, large number of clinical verifications has demonstrated that the Brain Natriuretic Peptide can be used in heart failure detection. Some relevant studies illustrate Plasma Brain Natriuretic Peptide can be affected by many factors, such as gender, age, environment of therapy, and so forth. This paper analyzes valid data of a clinical experiment, and finds out the influence of concomitant variables in diagnose of heart failure, then analyzes the outcome of rhNRG-1 on each individual. The phrase 'Brain Natriuretic Peptide' in the article specified N-terminal Prohormone of Brain Natriuretic Peptide (Nt-Pro BNP) in this dissertation. In this paper, the main analyzing method is Logistic Regression. It is used for estimating the Parameters of a qualitative model. The outcome, in other words, the probabilities is to describe the possible results of a single trial. By using this method, we could discuss the triggers of diseases and popularize it to other problems that concentrate on the cause. Moreover, the binary logistic model is for predicting a binary response based on one or more predictor variables. The main steps of this dissertation is data screening, missing values handling, descriptive statistics analyzing, then the Logistic Regression, and finally draw a conclusion.
\end{abstract}

Keywords: N-terminal Prohormone, Brain Natriuretic Peptide, Heart Failure, Biostatistics, Logistic Regression

\section{Introduction}

Biomedical statistics is a discipline, it is widely used in medical teaching and medical research. In the experimental implementation, data collection, data collation, results analysis [3]. The report writing and other links are related to statistical knowledge. Especially for a clinical trial with no statistical norms under the guidance of clinical trials, there is no set of strict control, on estimation of sample size and proper statistical analysis, the clinical trial is very difficult to be recognized.

In the clinical diagnosis of heart failure and NT proBNP level is widely used in the prediction and treatment of chronic heart failure and acute myocardial infarction (AMI), is also the only [7] [8] can be used for assessment of cardiac diastolic function indexes. Especially when exogenous BNP was introduced to treat heart failure, the Nt-ProBNP in the blood was not affected, so the left ventricular function and early treatment for heart failure could be accurately evaluated.

Multivariate logistic regression analysis can be used in many biomedical research processes. Such as death and death of the trial object, and the occurrence of the disease. However, these data are classified as dependent variable. The results of measurement of brain natriuretic peptide by classification of plasma brain natriuretic peptide concentration threshold will be the transformation of binary response variables, thus multiple logistic regression analysis.

\subsection{Purpose and Significance of Research}

This article is for the purpose of through logistic regression and other statistical methods, the collected data of analysis in the detection of patients with heart failure index covariates of brain natriuretic peptide (BNP) Yin positive effect and drug is given of NT proBNP in the diagnosis of heart failure in the overall evaluation of the model.

The significance of this study is that it can find the effect of Nt-ProBNP on the detection of heart failure covariates,

So as to reduce or control the effect of the co variable to the accuracy of the experiment in the next experiment, Provide 
reference for the future Nt-ProBNP biomedical experiments, and evaluate the effect of the drug to the individual.

\subsection{Research Background}

Heart failure is the final stage of many diseases, it can be divided into acute heart failure and chronic heart failure [13] [14]. In the past 30 years, despite the progress of heart failure diagnosis and treatment, but according to the Chinese cardiovascular disease report three years ago, China currently has about 4200000 patients with heart failure [13]. In addition, the number of patients with heart failure increased with the increase of population aging will increase significantly. As a result, the diagnosis of heart failure is a huge challenge for scientists and medical workers both in China and in the world.

September 2013 in our country in the official journal of the JACC [17] (Journal of the American College of Cardiology cardiovascular interventions is the American College of Cardiology (ACC), focusing on the heart peripheral vascular and cerebral vascular interventional research progress in areas such as): the control study, is to have listed a capsule [17] (domestic) multi center, randomized double blind, placebo controlled trial.

The study takes the NT-proBNP level as the main index to evaluate the therapeutic effect of this capsule on chronic heart failure.

Research results show that combined application of the capsule can significantly reduce the level of NT proBNP in patients with heart failure, in the standard anti heart failure treatment based on modified cardiac function and quality of life and so on.

It can be seen that the brain natriuretic peptide in the treatment of heart failure continues to develop, and has become mature in recent years.

However, there are still some problems in the diagnosis and treatment, such as the interference of the results of the association and so on.

\section{Clinical Trial Design}

Clinical trials in any healthy volunteers or patients of test drug research, it is through the contrast analysis of experimental group and control group results, confirm or reveal test with the effects of drugs, efficacy or adverse reactions of a scientific research. The main purpose of clinical trials is to ensure the efficacy and safety of the drugs in the future trials and trials. [17]. Clinical trial requirements and Clinical trial design process (see Figure 1).

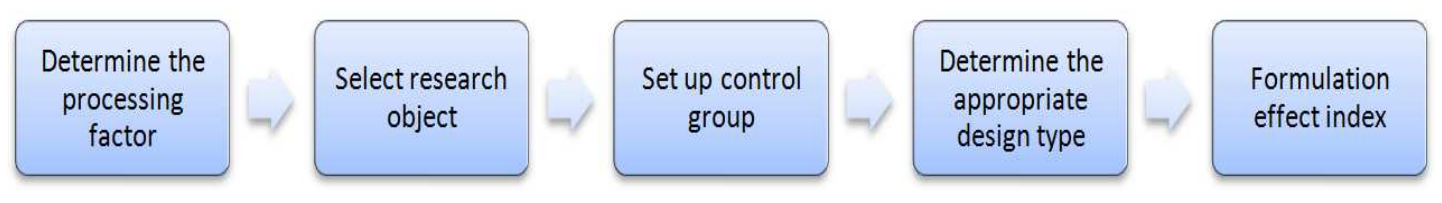

Figure 1. Clinical trial design process.

\subsection{Double-Blind Randomized Placebo-Controlled Trial}

\subsubsection{Multi Center Test}

Multicenter trial can be in a relatively short period of time to collect the required number of cases, and collected cases range more widely, so multi center clinical trial results for future applications more representative. But the impact factor is also more complex. Under the unified leadership of the organization, multi center test must follow a complete test plan to complete the whole experiment.

\subsubsection{Double Blind Test}

In clinical trials, we must follow the blind principle, and the blind method is mainly from single blind and double blind trials, [9]. In clinical trials, if the subjects knew that they ate the drug or were ineffective in the placebo, then the psychological factor may have positive and negative effects on the results of the experiment. Single blind trial is the subjects' specific content of the drug is unknown, and the researchers know that each subject specific drug use. Double blind trial means the researchers and subjects did not know each subject's grouping and receiving treatment, only after the trial can be blinded [9]. Because of the existence of the researchers in order to get good results, they will get a positive evaluation of the drug. Double blind trial can avoid the bias of the subjects and the researchers, which is to avoid the subjective deviation. This can improve the authenticity of the results significantly.

\subsubsection{Randomized Controlled Trial}

Randomization: in order to reduce the experimental error, the age of the two groups of subjects, health status, other factors to accept other treatment should be similar. To this end, researchers must follow the principle of randomization. In other words, all subjects were randomly assigned to different groups, which would enter a group that was completely randomly generated by numbers, rather than artificially selected for the group. Otherwise, the drug group can be selected for the lighter (heavier) patients, and the effect of the new drug group is too obvious (not significant). The control experiment theoretically assured the consistency of the other factors except the study factor except the experiment.

\subsubsection{Placebo}

The placebo had no effect and had no side effect. Generally, its appearance was the same as the real medicine, and it was generally used as the control group in clinical trials. [13].

\subsubsection{Parallel Grouping Design}

The patients were randomly assigned to a group of two treatment groups, each with a different treatment, the comparison of rhNRG-1 and placebo Placebo, respectively. 
The disadvantage of parallel control design is that the two groups of patients may have an imbalance related factors, and the required sample size, is two times cross design. The utility model has the advantages of obviously, subjects participated in time relative cross packet shorter, and therefore will not affect the basic to subjects of compliance, thereby avoiding some early abscission and loss to follow-up phenomenon [15].

\subsection{Nt-ProBNP Determination}

Biological characteristics of BNP 2.2.1 and Nt-ProBNP

The heart has the function of pumping blood, and is an endocrine organ [20].

Heart will adjust through constantly secretion of natriuretic peptide atrial ventricular pressure, at the same time; it can also adjust the endocrine function of the other. The BNP is an important member of the above mentioned natriuretic peptide in the family. [21] BNP is the two forms of BNP-32 and Nt-ProBNP, and Nt-ProBNP is the non active $\mathrm{N}$ amino terminal products. The clinical choice of Nt-ProBNP as a diagnostic drug, because compared with NT-ProBNP, BNP has many biological characteristics (advantage). [9][13]

Refer to the cardiac function classification, according to the New York heart disease association, namely NYHA, heart function is divided into I / II / III / IV. The classification principle is to determine the degree of activity of symptoms of heart failure to determine grade. [9] See Appendix two for the degree of concrete classification. Although this scheme is simple and easy, but only by patients subjective statement, and sometimes the patient's symptoms and objective examination and a big gap, and the difference between patients feeling and individual is also different.

The concentration threshold of plasma brain natriuretic peptide in this paper will be referred to the consensus [9] of the 2004 American Heart Disease Institute, as 400pg/L. That is, $\mathrm{BNP}>400 \mathrm{pg} / \mathrm{L}$ is considered as a disease.

\section{Data and Methods}

\subsection{Data Description and Data Processing}

In this paper, a series of clinical trial data is analyzed, the data comes from the author's biological Medicines Co. Note: the test has been blinded.

The data included 146 trials, of which were divided into group 1: placebo control group (Placebo), 67 case; group 2: drug group (rhNRG-1), 79 case.

There were 438 observations, each of the trials will receive three follow-up, respectively, at the beginning of the trial for zeroth days, thirtieth days, ninetieth days. Gender: 1- male, 0female.

Data loss processing. The data of heart failure index (sujid) was 4206 , and the object was group 1, and it was decided to delete the missing value, so the data was invalid. So the observation of effective data is 435 . In which the 1 groups of the 193 observation, the group of 237 containing 2 observations. In the next logistics regression analysis, the heart failure index was divided into two groups (sick and not sick). The Convention had a positive case (positive, 1), and had no negative case (negative, 0 ).

\subsection{Logistic Regression Principle}

The regression analysis is mainly based on a set of independent variables (predictor) to predict the statistical method of a (multiple) dependent variable (response variable).It can also be used to evaluate the effect of the predictor variables on the response variables [17]. In most practical problems, the factors that affect the dependent variable are not only one, but more, which need to be analyzed by using multiple regression method. Regression Logistic is one of the most widely used methods for multivariate statistical analysis.

Logistic regression is the case of the type of variable, such as " 0,1 ".Because the variable $\mathrm{X}$ is the result of the $\mathrm{Y}$ of a set of independent variables, the assignment rules are: The probability of -1 is represented by $\mathrm{P}$. The negative example results -0 , the probability $\mathrm{Q}=(1-\mathrm{P})$ to express.

Logistic regression model:

$$
\ln \left(\frac{P}{Q}\right)=\ln \left(\frac{P}{1-P}\right)=\beta_{0}+\beta_{1} X_{1}+\beta_{2} X_{2}+\cdots+\beta_{m} X_{m}
$$

In statistics, $\ln (\mathrm{P} / \mathrm{Q})$ is called the $\mathrm{P}$ conversion or Logit conversion, that is, Logit $(\mathrm{P})$. The resulting regression equation is Logistic regression equation. We can get from (1):

$$
P=\frac{e^{\beta_{0}+\beta_{1} X_{1}+\beta_{2} X_{2}+\cdots+\beta_{m} X_{m}}}{1+e^{\beta_{0}+\beta_{1} X_{1}+\beta_{2} X_{2}+\cdots+\beta_{m} X_{m}}}
$$

The logistic regression model derived from sample estimates:

$$
\begin{gathered}
\ln \left(\frac{P}{1-P}\right)=b_{0}+b_{1} X_{1}+b_{2} X_{2}+\cdots+b_{m} X_{m} \\
P=\frac{e^{b_{0}+b_{1} X_{1}+b_{2} X_{2}+\cdots+b_{m} X_{m}}}{1+e^{b_{0}+b_{1} X_{1}+b_{2} X_{2}+\cdots+b_{m} X_{m}}}
\end{gathered}
$$

Where $\mathrm{P} / \mathrm{Q}$ is odds, the ratio of odds is $\mathrm{P} /(1-\mathrm{P})$ is called ratio (OR).

The probability of occurrence of the I (odds) was Pi/Qi, Then:

$$
\ln \left(\frac{P_{i}}{Q_{i}}\right)=b_{0}+b_{1} X_{i 1}+b_{2} X_{i 2}+\cdots+b_{m} X_{i m}
$$

The incidence of the 1 was $\mathrm{Pl} / \mathrm{Q} 1$,

Then:

$$
\ln \left(\frac{P_{l}}{Q_{l}}\right)=b_{0}+b_{1} X_{l 1}+b_{2} X_{l 2}+\cdots+b_{m} X_{l m}
$$




$$
\begin{array}{r}
\ln \left(\frac{P_{i} / Q_{i}}{P_{l} / Q_{l}}\right)=\ln (O R)=b_{1}\left(x_{i 1}-x_{l 1}\right)+b_{2}\left(x_{i 2}-x_{l 2}\right)+\cdots+b_{m}\left(x_{i m}-x_{l m}\right) \\
\ln \left(\frac{P_{i} / Q_{i}}{P_{l} / Q_{l}}\right)=\ln (O R)=b_{1}\left(x_{i 1}-x_{l 1}\right)+b_{2}\left(x_{i 2}-x_{l 2}\right)+\cdots+b_{m}\left(x_{i m}-x_{l m}\right)
\end{array}
$$

$$
\begin{aligned}
& \text { If } \mathrm{xj} \text { is assigned to } x_{j}=\left\{\begin{array}{l}
1, \text { Exposure } \\
0, \text { Non exposure }
\end{array}\right. \text { then } \\
& O R_{j}=\exp \left(b_{j}\right)=e^{b_{j}}
\end{aligned}
$$

Where :

when $b j=0, O R j=1$, The factor $x j$ does not work for the disease;

When $b j>0, \quad O R j>1$, ; The factor $x j$ is a risk factor

When $b j<0, \quad O R j<1$, The factor $x j$ is a protective factor

For the chronic disease with particularly low morbidity, OR can be used as the approximate estimation of RR because of $\mathrm{P}<<1$.

$$
O R=\frac{P_{1} /\left(1-P_{1}\right)}{P_{0} /\left(1-P_{0}\right)} \approx \frac{P_{1}}{P_{0}}=R R
$$

Above, The reasons for the epidemiological investigation of Regression Logistic were revealed. Its advantage is that the regression coefficient of a factor is obtained. The approximate estimate of relative risk under different levels is obtained. So this paper uses Regression Logistic as the core analysis method.

\section{Data Analysis}

\subsection{Descriptive Statistics}

After the completion of the missing values, all 435 observations were selected (see Table 1).

Table 1. Case Processing Summary.

\begin{tabular}{llllllll}
\hline & \multicolumn{3}{l}{ Cases } & & & & \\
\cline { 2 - 7 } & \multicolumn{2}{l}{ Included } & Excluded & \multicolumn{2}{l}{ Total } & \\
\cline { 2 - 7 } & N & Percent & N & Percent & N & Percent \\
\hline $\begin{array}{l}\text { drug * ntProbnP * } \\
\text { visitnum }\end{array}$ & 435 & $100.00 \%$ & 0 & $0.00 \%$ & 435 & $100.00 \%$ \\
\hline
\end{tabular}

Next, the experimental group and the control group were described statistically, The mean, variance and standard deviation of the plasma Nt-ProBNP concentrations in the two groups of subjects, $95 \%$ confidence interval and upper bound lower bound (see Table 2).

Table 2. Descriptive drug.

\begin{tabular}{lllllll}
\hline \multirow{2}{*}{ Nt-proBNP } & \multirow{2}{*}{$\mathbf{N}$} & Mean & Std. Deviation & \multirow{2}{*}{ Std. Error } & & \multicolumn{2}{c}{ 95\% Confidence Interval for Mean } \\
\cline { 6 - 7 } & & & & & Lower Bound & Upper Bound \\
\hline 1 & 79 & 1618.97 & 1317.619 & 160.973 & 1297.58 & 1940.36 \\
Total & 146 & 1716.49 & 1709.074 & 192.286 & 1415.68 & 2181.31 \\
\hline
\end{tabular}

\subsection{ANOVA Variance Analysis}

Analysis of variance for treatment groups (see Table 3). From the results of the "single factor ANOVA" analysis results, significantly 0.484 , from $0.484>0.05$, it can be concluded that the treatment group had no significant effect on the Nt-ProBNP. So the next regression logistic covariates will not include the treatment group.

Table 3. ANOVA (drug).

\begin{tabular}{llllll}
\hline & $\begin{array}{l}\text { Sum of } \\
\text { Squares }\end{array}$ & df & $\begin{array}{l}\text { Mean } \\
\text { Square }\end{array}$ & F & Sig. \\
\hline Between Groups & 1168400.559 & 1 & 1168401 & 0.491 & 0.484 \\
Within Groups & $3.42 \mathrm{E}+08$ & 144 & 2377895 & & \\
Total & $3.44 \mathrm{E}+08$ & 145 & & & \\
\hline
\end{tabular}

\subsection{Logistic Regression Analysis}

The number of experiments was 438 times and the number of events was 435 times.. Analysis of response variables: the basic information of the model was given, the disease occurred 386 times and no 52 times.

In the model, the stepwise selection method is adopted.,

Meet the convergence criteria $(\mathrm{GCONV}=1 \mathrm{E}-8)$, Log L -2 (ratio L: likehood) and Score is used to detect whether the independent variables are significant indicators. SC (Schwarz criterion and AIC (Akaike information criterion) two information criterion is used to compare different models, with smaller values, model is better, so - $2 \log \mathrm{L}$ and score corresponding to the $\mathrm{p}$ value is also getting smaller and smaller.

Table 4. Stepwise selection process.

\begin{tabular}{llll}
\hline Steps & $\begin{array}{l}\text { Step1:age get } \\
\text { in: }\end{array}$ & $\begin{array}{l}\text { Step2:site ID get } \\
\text { in }\end{array}$ & $\begin{array}{l}\text { Step3:gender get } \\
\text { in }\end{array}$ \\
\hline $\mathrm{P}(\mathrm{L})$ & $\mathrm{P}(\mathrm{L})<0.05$ & $\mathrm{P}(\mathrm{L})<0.05$ & $\mathrm{P}(\mathrm{L})<0.05$ \\
$\mathrm{P}($ score $)$ & $\mathrm{P}($ score $)<0.05$ & $\mathrm{P}($ score $)<0.05$ & $\mathrm{P}($ score $)<0.05$ \\
& Significant & Significant & Significant \\
Significant & explanatory & explanatory & explanatory \\
& variable & variable & variable \\
\hline
\end{tabular}


Estimation of model parameters (see Table 5).

Table 5. Maximum Likelihood Estimation.

\begin{tabular}{llllll}
\hline Parameter & Freedom & $\begin{array}{l}\text { Estimate } \\
\text { value }\end{array}$ & $\begin{array}{l}\text { Standard } \\
\text { error }\end{array}$ & $\begin{array}{l}\text { Wald } \\
\text { card }\end{array}$ & $\begin{array}{l}\text { Pr> } \\
\text { card }\end{array}$ \\
\hline Intercept & 1 & 2.2856 & 1.1304 & 4.088 & 0.0432 \\
ntprobnp & 1 & 0.1128 & 0.3202 & 0.1242 & 0.7246 \\
visitnum & 1 & -0.00877 & 0.00419 & 4.3764 & 0.0364 \\
siteid & 1 & -0.0372 & 0.01 & 13.7645 & 0.0002 \\
gender & 1 & -1.4944 & 0.5675 & 6.9351 & 0.0085 \\
age & 1 & 0.069 & 0.014 & 24.159 & $<.0001$ \\
\hline
\end{tabular}
be:

The form of the modified Logistic regression equation can

Logit $(\mathrm{P})=2.2856+0.1128-0.00877-0.0372 *$ siteid$1.4944 *$ gender $+0.0690 *$ age

The point estimate of each parameter (see Table 6)
Table 6. the odds ratio estimate.

\begin{tabular}{lllc}
\hline Effect & Point estimate & \multicolumn{2}{c}{ 95\% Wald confidence limit } \\
\hline ntprobnp & 1.119 & 0.598 & 2.097 \\
visitnum & 0.991 & 0.983 & 0.999 \\
siteid & 0.963 & 0.945 & 0.983 \\
gender & 0.224 & 0.074 & 0.682 \\
age & 1.071 & 1.042 & 1.101 \\
\hline
\end{tabular}

\subsection{Two Classification Model Performance Evaluation}

Receiver operating characteristic curve (ROC curve) (see Figure 2), Sensitivity and specificity were combined with the ROC graph. It is also an important graph of the model compared to the baseline (Fig. 45 degree curve).The clinical accuracy of the method can be observed by it.Can be seen from the picture, $\mathrm{AUC}=0.8048$, it is proved that this model is better than random guesses. The model properly set the threshold will be predictive value; in other words, the accuracy of the model is higher.

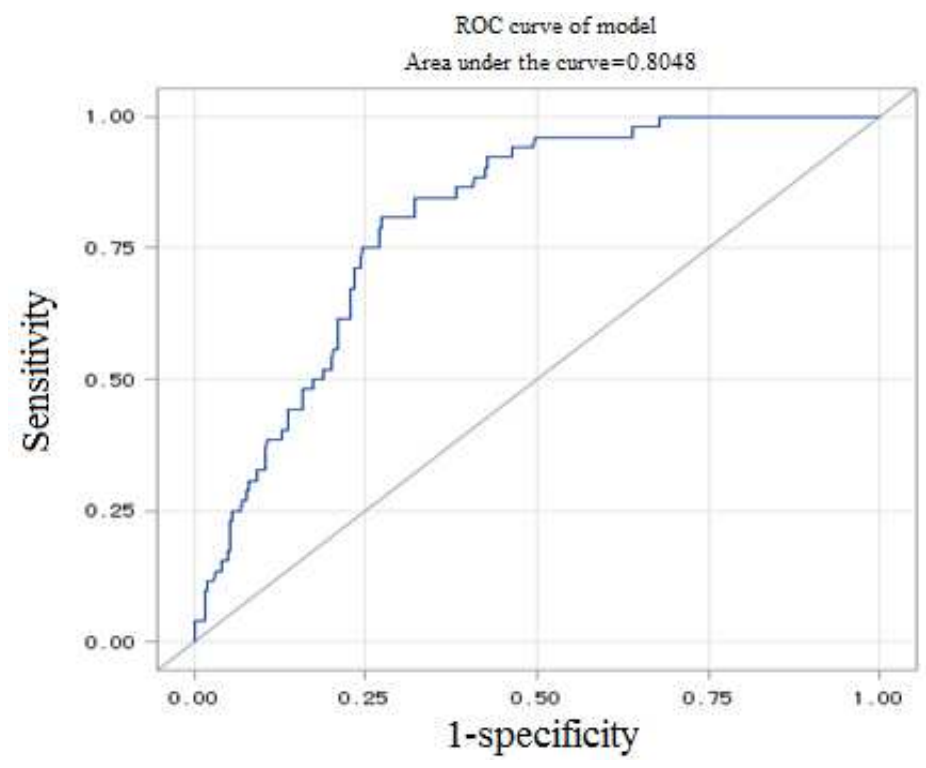

Figure 2. ROC diagram.

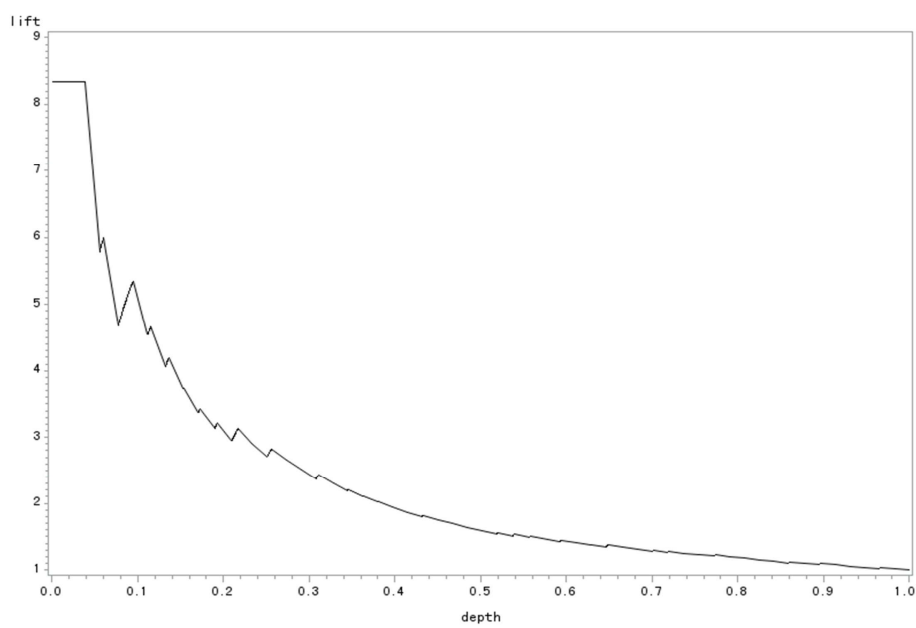

Figure 3. lift diagram. 
Lift curve graph as model promoting force forecast, It reveals the predictive power of the model to "better" than the model without using the model. That is, the lift chart shows the effect of the logistic model, the higher the index, the better. In the lift diagram, Depth is predicting the proportion of cases, as the threshold decreases, the more steep as expected; Thus, the model is more general, (see Figure 3).

\section{Concludes and Discusses}

\subsection{Research Content Summary}

Through the statistical analysis of clinical brain natriuretic peptide in heart failure detection test data, it is concluded that the conclusion of the trial forecast values and the existing level has a relatively strong correlation and regression model has a strong predictive ability. The percentage of the agreement was $80.2 \%$ and the percentage of the inconsistency was $19.4 \%$, see Table 7. The prediction accuracy of the effect of the drug rhNRG-1, showing the individual adaptation, then can be targeted for the treatment and medication for patients.

Table 7. Connection of Prediction Probability and Observing Respond.

\begin{tabular}{llll}
\hline Percentage of consistency & 80.2 & Somers D & 0.608 \\
Percentage of inconsistency & 19.4 & Gamma & 0.61 \\
Knot value percentage & 0.4 & Tau-a & 0.128 \\
weight & 19916 & c & 0.804 \\
\hline
\end{tabular}

\subsection{Research Prospects}

$\mathrm{Nt}$-proBNP is developing rapidly in diagnosis and treatment of heart failure, and is the most common drug in clinic.. However, because the detection method is different, the reference range of the normal values of Nt-proBNP is different, and different people also have different reference values.. For example, with the increase of age, the concentration of BNP in plasma increased, and the female ratio was slightly higher than male [20]. These factors can affect the treatment of heart failure patients, so it is a field of further research and analysis to improve the diagnostic accuracy and eliminate the interference.

\section{References}

[1] Safety and feasibility of using serial infusions of nesiritide for heart failure in an out patient setting (from the FUSION I trial). Yancy CW et al. American Journal of Cardiology. 2004 1; 94(5):595-601.

[2] Liu Xin, Tong Wang. Clinical significance and research progress of brain natriuretic peptide in plasma. International Journal of medical testing medicine 2009.3. 30 No. 3.

[3] Shi Chenghu, Hu Fengtao. Clinical application of BNP brain natriuretic peptide [R]. Hubei County, Huanggang City, Huangmei Province, the people's Hospital, laboratory. 1671-8194 (2013) 12-0470-02.

[4] Chen Xiaofei. BNP value analysis of cardiac function classification and prognosis in patients with chronic heart failure [J]. Modern diagnosis and treatment, 2012.Dec23 (12): 2122-2123.

[5] Liang Xiaoying. The influence factors of BNP and NT-proBNP detection threshold concentration [J]. Chinese Practical Medicine, December 2007 second volume thirty-sixth: 161-163.

[6] Zheng Siju. B type natriuretic peptide as indicators of the significance of determination of heart dysfunction [J]. Shenyang army medicine, May 2005 eighteenth volume third: 209-211.

[7] Han Jing. History, status quo and future of heart failure drug therapy [C]. 2012.4.12.

[8] Yang Yurong. Clinical significance of B natriuretic peptide in heart failure $[\mathrm{J}]$. Chinese modern drug application, August 2010 fourth volume fifteenth: $39-40$.

[9] Zhang Xiaojun. The effect of BNP on the cardiac function classification in patients with heart failure J Lab Diagn March, Vol16, 2012, No.3:472-474. Chin.

[10] Xiao Yipin. To investigate the clinical analysis of surgical treatment for colon cancer [J], Chinese health nutrition clinical medicine, 2013.12 (on):6961-6962.

[11] Zhang Xiaohui, Essien, Huo Xinghui, Li Lu. Discussion on the standardization of clinical trial management for drugs [J], Changchun Institute of traditional Chinese Medicine 20033. 